Draft Version June 28, 2021

Preprint typeset using $\mathrm{LAT}_{\mathrm{E}} \mathrm{X}$ style emulateapj v. 12/16/11

\title{
POPULATION SYNTHESIS OF BLACK HOLE BINARIES WITH NORMAL-STAR COMPANIONS: I. DETACHED SYSTEMS
}

\author{
YONG SHAO ${ }^{1,2}$ AND XIANG-DONG Li $^{1,2}$ \\ ${ }^{1}$ Department of Astronomy, Nanjing University, Nanjing 210046, People's Republic of China; shaoyong@nju.edu.cn and \\ ${ }^{2}$ Key laboratory of Modern Astronomy and Astrophysics (Nanjing University), Ministry of Education, Nanjing 210046, People's \\ Republic of China; lixd@nju.edu.cn \\ Draft version June 28, 2021
}

\begin{abstract}
Optical observations of normal-stars in binary systems with massive unseen objects have been proposed to search for candidate black holes $(\mathrm{BHs})$ and provide a direct measurement of their dynamical masses. In this paper, we have performed binary population synthesis calculations to simulate the potential population of detached binaries containing $\mathrm{BHs}$ and normal-star companions in the Galaxy. We focus on the influence of the $\mathrm{BH}$ progenitors. In the traditional model, BHs in binaries evolve from stars more massive than $\sim 25 M_{\odot}$. However, it is difficult for this model to produce BH low-mass $\mathrm{X}$-ray binaries. Recent investigations on massive star evolution suggest that the $\mathrm{BH}$ progenitors may have masses as low as $\sim 15 M_{\odot}$. Based on this model, we provide the expected distributions of various parameters for detached $\mathrm{BH}$ binaries with normal-star companions, including the component masses, the orbital parameters of the binary systems, the radial velocity semi-amplitudes, and the astrometric signatures of the optical companions. Our calculations show that there are more than thousands of such detached binaries in the Galaxy, and hundreds of them are potentially observable systems with luminous companions brighter than $20 \mathrm{mag}$. In addition, detached $\mathrm{BH}$ binaries are dominated by those with main-sequence companions and only a few percent of them are expected to have giant companions.
\end{abstract}

Subject headings: binaries: general - stars: black holes - stars: evolution

\section{INTRODUCTION}

It is believed that there are hundreds of millions of stellar-mass black holes (BHs) in the Galaxy (van den Heuvel 1992; Brown \& Bethe 1994, Timmes et al. 1996). More than forty years has passed since the discovery of the first BH in Cygnus X-1 (Bolton 1972; Webster \& Murdin 1972). To date, however, only two dozen of BHs have been dynamically confirmed. The majority of them are discovered in X-ray binaries (Remillard \& McClintock 2006; Casares \& Jonker 2014), in which the BH is accreting material from its companion star and emitting $\mathrm{X}$-ray radiation. According to binary evolution theories, quite a number of binary systems are likely to host a quiescent $\mathrm{BH}$ orbiting its normal-star ${ }^{1}$ companion, prior to the $\mathrm{X}$-ray binary phase, since the $\mathrm{X}$-ray radiation due to $\mathrm{BH}$ accretion is too weak to be detected.

A promising approach based on radial velocity searches has been proposed to discover BHs in binary systems for decades (Guseinov \& Zel'dovich 1966, Trimble \& Thorne 1969). Until recently, dynamical searches of optical companions are broadly used to identify BHs in binary systems. In the globular cluster NGC 3201, Giesers et al. (2018) found a main-sequence (MS) turn-off star orbiting an unseen component with large radial velocity variations. A deep analysis of the orbital parameters indicated that the unseen object is a potential $\mathrm{BH}$ with a minimum mass of $\gtrsim 4 M_{\odot}$. Based on the spectroscopic and photometric study of the binary AS 386, Khokhlov et al. (2018) revealed that the binary system has a circular orbit with a period of $\sim 131$ days, and contains

\footnotetext{
${ }^{1}$ In this work, a normal-star specifically refers to a star staying at the main-sequence or (super)giant stage.
}

a B-type star of mass $7 \pm 1 M_{\odot}$. Based on the absence of any traces of the secondary component, whose mass is larger than $7 M_{\odot}$, Khokhlov et al. (2018) suggested that it is most likely a BH. By combining radial velocity measurement with photometric variability data, Thompson et al. (2019) discovered a candidate $\mathrm{BH}$ orbiting a $\sim 3 M_{\odot}$ giant star with an orbital period of $\sim 83$ days. Since BHs discovered in such detached binaries are not subject to the effect of possible mass accretion, a large sample in the future can give a clear clue of the BH mass function and test the theories of binary evolution and the supernova (SN) mechanism.

According to some related surveys, the prospect of hunting $\mathrm{BHs}$ in binaries with visible normal-stars has already been investigated. For the astrometric satellite Gaia over its five year mission, several groups (Breivik et al. 2017; Mashian \& Loeb 2017; Yamaguchi et al. 2018; Yalinewich et al. 2018) predicted that dozens or thousands of binaries with a $\mathrm{BH}$ component may be discovered. Using the photometric data of Transiting Exoplanet Survey Satellite, Masuda \& Hotokezaka (2018) discussed the potential of identifying BHs with normalstar companions on tight but detached orbits. Based on spectroscopic observations of Large sky Area MultiObject fiber Spectroscopic Telescope, Gu et al. (2019) proposed a method to search for stellar-mass BH candidates in binaries with giant companions.

Stellar evolution predicts that BHs originate from massive stars with masses $\gtrsim 20-25 M_{\odot}$ (Woosley \& Weaver 1995; Fryer et al. 2012). The standard formation scenario for $\mathrm{BH}$ binaries with initially a low-mass companion usually involves a common envelope (CE) phase (see a review by Ivanova et al. 2013) in which the spiral-in 
of the low-mass secondary causes the ejection of the envelope of the primary (BH's progenitor). However, it was pointed out that the primary's envelope is too massive for a low-mass secondary to strip off (Podsiadlowski) et al. 2003), probably resulting in the binary merge. In view of the $\mathrm{CE}$ phase, compact $\mathrm{BH}$ binaries with lowmass companions can be formed only if adopting high values for the binding energy parameter of the primary envelope (Podsiadlowski et al. 2003) or for the CE ejection efficiency (Kiel \& Hurley 2006; Yungelson \& Lasota 2008). Another solution to this problem is adopting relatively small masses for the $\mathrm{BH}$ progenitors (Wang et al. 2016a). According to the argument of Kochanek (2014), stars with initial masses $\gtrsim 17 M_{\odot}$ may experience failed explosions and evolve to BHs. Wang et al. (2016a) showed that short orbital-period BH low-mass $\mathrm{X}$-ray binaries can be effectively produced through the standard CE scenario if most BHs are born in failed SNe. It has been long known that the pre-SN core structure of a massive star greatly determines the final fate of either explosion or implosion (Burrows et al. 1995). Recently, some numerical simulations (e.g., $\mathrm{O}^{\prime}$ Connor \& Ott 2011; Ugliano et al. 2012, Pejcha \& Thompson 2015; Ertl et al. 2016 Sukhbold et al. 2016) showed that the landscape of neutrino-driven explosions is strongly manipulated by the final core structure of massive stars, and there is no clean threshold to separate the outcomes of either neutron stars (NSs) or BHs. Stars with masses $\sim 15-21 M_{\odot}$ still have a possibility to eventually implode to a $\mathrm{BH}$; on the contrary, a star massive than $20 M_{\odot}$ may successfully explode to become an NS (Raithel et al. 2018).

In this work, we perform binary population synthesis (BPS) calculations to simulate the Galactic population of $\mathrm{BH}$ binaries with normal-star companions. The main goal of this work is to estimate the number and parameter distribution of $\mathrm{BH}$ binaries that can be potentially discovered through optical observations of the normalstar companions. We here only consider the detached systems without the occurrence of Roche lobe overflow. In a forthcoming study, we will discuss the population of mass-transferring $\mathrm{BH}$ systems (i.e., X-ray binaries) in the Galaxy. The remainder of this paper is organized as follows. In Section 2, we introduce the BPS method, according to which we can generate a large number of $\mathrm{BH}$ binaries with normal-star companions. We present the calculated results and discussions in Section 3. Finally we conclude in Section 4.

\section{METHOD}

The X-ray emission in detached systems should be very weak and even undetected due to little accretion onto the BHs. The BH masses in such binaries, however, can still be measured from the motions of the optical companions. In order to obtain the potential population of detached $\mathrm{BH}$ binaries with a normal-star companion in the Galaxy, we employ the population synthesis code $B S E$ originally developed by Hurley et al. (2002). With $B S E$ we can simulate the evolution of millions of binary stars with different initial parameters. The binary evolution is assumed to start from primordial binaries with two zero-age MS stars, and the subsequent evolution will be subject to many physical processes, e.g. mass and angular momentum transfer, $\mathrm{CE}$ evolution, $\mathrm{BH}$ formation and natal kicks. A detail modification of the code has been made by Shao \& Li (2014), here we notice some important points in the following.

We only consider the detached $\mathrm{BH}$ binaries formed through the evolution of isolated binaries, that is, systems formed through dynamical interactions in global clusters are not included. During the evolution of the primordial binaries, the primary star first evolves to expand, then transfers mass to the secondary. Thus one first needs to determine whether the mass transfer is dynamically stable. This is critically dependent on the mass ratio of the primary and secondary stars and the mass accretion efficiency of the secondary star. Shao \& Li (2014) built three mass transfer modes to deal with the mass exchange between binary components, among which the rotation-dependent mode (assuming the accretion efficiency of the secondary to be dependent on its rotating velocity) appears to better reproduce the observed parameter distribution of Galactic binaries including BH-Be star systems (Shao \& Li 2014), Wolf-Rayet star-O-type star systems (Shao \& Li 2016) and NS-NS systems (Shao \& Li 2018). Thus we adopt the rotationdependent mass transfer mode, in which the accretion efficiency of the secondary can be as low as $<0.2$ and the corresponding mass ratio of the primary to the secondary for stable mass transfer can reach $\sim 6$ (Shao \& Li 2014). Compared with the traditional conservative mass transfer, this allows a much larger parameter space for stable mass transfer in the primordial binaries.

The binaries experiencing dynamically unstable mass transfer will go into CE evolution. The orbital energy of the embedded binary is used to eject the envelope. We adopt the standard energy conservation equation (Webbink 1984) to deal with the orbital decay, taking the binding energy parameter $\lambda$ calculated by $\mathrm{Xu} \& \mathrm{Li}(2010)$, and the $\mathrm{CE}$ ejection efficiency $\alpha_{\mathrm{CE}}$ is assumed to be unity ${ }^{2}$. After $\mathrm{CE}$ evolution, the remnant binaries may survive if they do not merge during the spiral-in stages. Stellar mass loss rates of Hurley et al. (2000) are employed, except for hot OB stars, for which we apply the simulated rates of Vink et al. (2001). When the entire envelope of the primary star is stripped due to binary interactions, we reduce the mass loss prescription of Hamann et al. (1995) by a factor of 2 for helium stars (Kiel \& Hurley 2006).

At their formation, the BHs may be imparted a natal kick, resulting in eccentric orbits or even disruption of the binary systems. The SN processes play a vital role in determining both the magnitude of the kick velocities and the weight of the $\mathrm{BH}$ masses. In order to account for the $\sim 2-5 M_{\odot}$ gap between NS and BH masses, Fryer et al. (2012) proposed the rapid SN mechanism ${ }^{3}$ assuming that the final mass of a compact object is contributed by the proto-compact object and the fallback material. With

\footnotetext{
${ }^{2}$ Recently Fragos et al. (2019) simulated the inspiral of a $1.4 M_{\odot}$ NS inside the envelope of a $12 M_{\odot}$ red supergiant star and suggested a very high $\alpha_{C E}$-equivalent efficiency of $\approx 5$. Also, Mapelli \& Giacobbo (2018) showed that the cosmic merger rates predicted tor $\mathrm{NS}-\mathrm{NS}$ binaries are consistent with LIGO-Virgo estimations when adopting $\alpha_{\mathrm{CE}}=5$ in population synthesis models. If the $\alpha_{\mathrm{CE}}$ is indeed higher than unity, it could help resolve the formation problem of BH low-mass X-ray binaries (Podsiadlowski et al. 2003 Kiel \& Hurley 2006 Yungelson \& Lasota 2008).

3 Note that the other method of the delayed SN mechanism in Fryer et al. (2012) allows the formation of low mass BHs, so is not included in our calculations.
} 
this mechanism stars with masses $\gtrsim 20 M_{\odot}$ could evolve to BHs. More recently, Sukhbold et al. (2016) suggested that stars with masses as low as $15 M_{\odot}$ have a chance to implode to be a $\mathrm{BH}$, and the average likelihoods for stars with initial masses of $15-40 M_{\odot}$ and $45-120 M_{\odot}$ were respectively 0.574 and 0.656 (Raithel et al. 2018). In this case the $\mathrm{BH}$ masses were directly obtained as the remnant masses of pre-SN stars, or the combined masses of the helium core and a small fraction $(\lesssim 0.1)$ of the envelope if exists. In our simulations we consider both prescriptions to deal with the $\mathrm{BH}$ masses. The first one is $M_{\mathrm{BH}}=0.9\left(M_{\text {proto }}+M_{\mathrm{fb}}\right)$ where $M_{\text {proto }}$ and $M_{\mathrm{fb}}$ are respectively the masses of the proto-compact object and the fallback material. The other one is $M_{\mathrm{BH}}=0.9 M_{\text {rem }}$, where $M_{\text {rem }}$ is the pre-SN remnant masses. Note that the pre-SN primaries in our BPS calculations are always helium stars without any hydrogen envelope due to binary interactions, and the stars with $M_{\text {rem }}=5 M_{\odot}$ have initial masses close to $15 M_{\odot}$. Following the suggestion of Raithel et al. (2018), we assume that all pre-SN primaries with masses larger than $5 M_{\odot}$ have a probability of 0.6 to form BHs.

For BH natal kicks in the former situation, we use the NS kick velocity reduced by a factor of $\left(1-f_{\mathrm{fb}}\right)$, where $f_{\mathrm{fb}}$ is the fallback material fraction (denoted as Model A). Here the kick velocity for NSs is assumed to follow a Maxwellian distribution with a dispersion of $\sigma_{\mathrm{k}}=265 \mathrm{~km} \mathrm{~s}^{-1}$ (Hobbs et al. 2005). In the later situation, the $\mathrm{BH}$ formation does not involve the fallback process, we explore three variations of the $\mathrm{BH}$ natal kicks: (1) using the NS kick velocity reduced by a factor of $\left(3 M_{\odot} / M_{\mathrm{BH}}\right)$ (denoted as Model B) obeying a Maxwellian distribution with a dispersion of $\sigma_{\mathrm{k}}=150 \mathrm{kms}^{-1}$ (denoted as Model C); (3) adopting a Maxwellian distribution with a smaller dispersion of $\sigma_{\mathrm{k}}=50 \mathrm{~km} \mathrm{~s}^{-1}$ (denoted as Model D).

We simulate the evolution of the primordial binaries by setting the initial parameters as follows. For the primary stars, we use the initial mass function (IMF) given by Kroupa et al. (1993). For the secondary stars, we assume a flat mass ratio distribution between 0 and 1 . The distribution of initial orbital separations is assumed to be logarithmically flat (Abt 1983). We set the initial orbits of all binaries to be circular, as the outcome of the interactions of systems with the same semilatus rectum is almost independent of eccentricity (Hurley et al. 2002). We assume all stars are initially in binaries. 'The primordial binaries are thought to follow the distribution of stars in the Galaxy and the $\mathrm{BH}$ binaries are simply assumed to be close to their birth locations without considering possible motions. The initial metallicity of stars is set to be 0.02 . Considering the star formation history of the Galaxy, we adopt a constant star formation rate of $3 M_{\odot} \mathrm{yr}^{-1}$ over the past $10 \mathrm{Gyr}$ period (Smith et al. 1978, Diehl et al. 2006; Robitaille \& Whitney 2010).

If a primordial binary evolves through a phase that is identified to be a detached BH system, then such a binary makes a contribution to the birthrate of the specific type

\footnotetext{
4 The value of 0.9 denotes an efficiency to convert baryonic to gravitational masses (e.g., Timmes et al. 1996).

5 In this model, the kick velocities are assumed to be inversely proportional to the $\mathrm{BH}$ masses, and the minimum $\mathrm{BH}$ mass is set to be $3 M_{\odot}$ (Wang et al. 2016a).
}

of detached BH system. We follow the method of Hurley et al. (2002) to calculate the birthrate for each type of detached BH binaries generated by our BPS calculations, which depends on the star formation rate of the Galaxy and the initial parameters of the primordial binaries.

\section{RESULTS AND DISCUSSIONS}

\subsection{Mass spectrum of newborn $\mathrm{BHs}$}

In Figure 1 we show the distributions of $\mathrm{BH}$ mass $M_{\mathrm{BH}}$ as a function of the initial mass $M_{\mathrm{i}}$ of the primary star. The four panels correspond to the adopted models $\mathrm{A}-\mathrm{D}$. In each panel, the black dots (with a number of $5 \times 10^{3}$ ) represent the output of the BPS calculations, and the red curve the $\mathrm{BH}$ mass spectrum for single star evolution. In all the four panels, there is a dip in the red curve around $50 M_{\odot}$, which is caused by the very strong winds from luminous blue variables. For stars more massive than $50 M_{\odot}$, the $\mathrm{BH}$ mass spectra in both single and binary evolution cases are broadly consistent with each other; for stars less massive than $50 M_{\odot}$, the BHs evolved from single stars are generally more massive than those from binary evolution, since the mass transfer in the primordial binaries decreases the mass of the primary stars. However, in Model A, there is an abrupt step at $23 M_{\odot} \lesssim M_{\mathrm{i}} \lesssim 28 M_{\odot}$ in the case of single star evolution and at $25 M_{\odot} \lesssim M_{\mathrm{i}} \lesssim 40 M_{\odot}$ in the case of binary evolution, corresponding to the process of direct collapse in the rapid SN mechanism (Fryer et al. 2012; See also Spera et al. 2015). We obtain $M_{\mathrm{BH}} \sim 5-16 M_{\odot}$ in all the models. Spera et al. (2015) used the population synthesis code $S E V N$ to track the stellar evolution and obtained the mass spectrum of stellar-mass BHs. Applying the rapid SN model, Spera et al. (2015) derived the masses of $\mathrm{BHs}$ in the range of $\sim 6-25 M_{\odot}$. This difference may originate from the different treatment of stellar winds.

\subsection{Formation of incipient BH binaries}

An incipient $\mathrm{BH}$ binary is a binary system just after the $\mathrm{BH}$ formation. Usually the companion is an MS star. Based on the BPS calculations, we create several $10^{5}$ incipient $\mathrm{BH}$ binaries in each model, then pick out the systems with orbital periods less than $10^{3}$ days. Figure 2 shows the calculated birthrate distributions of the incipient $\mathrm{BH}$ binaries in the companion mass $M_{\mathrm{c}}$ vs. orbital period $P_{\text {orb }}$ plane in different models. The colors in each pixel are scaled according to the weight of the corresponding birthrates. It is clearly seen that the incipient $\mathrm{BH}$ binaries with low-mass $\left(\lesssim 5 M_{\odot}\right)$ companions are hardly produced in Model A. The initial masses of the $\mathrm{BH}$ progenitors in this model are larger than $\sim 25 M_{\odot}$, so low-mass secondaries are not able to eject the primary's envelopes during CE evolution (see also Podsiadlowski et al. 2003, Wang et al. 2016a), leading the binary system to merge to be a single star. Almost all of the incipient $\mathrm{BH}$ systems produced in Model A are the descendants of the primordial binaries experiencing stable mass transfer during the evolution. The companion masses distribute in the range of $\sim 5-50 M_{\odot}$ and the orbital periods of $\sim 1-1000$ days, the overall birthrate in the Galaxy is about $9.0 \times 10^{-5} \mathrm{yr}^{-1}$. In Model B, the minimum companion mass is $\sim 0.7 M_{\odot}$, and the total birthrate of incipient $\mathrm{BH}$ systems is $\sim 6.0 \times 10^{-5} \mathrm{yr}^{-1}$. This is because the initial masses of the $\mathrm{BH}$ progenitors in this model decrease to $\sim 15 M_{\odot}$ (Raithel et al. 2018), so binaries with 

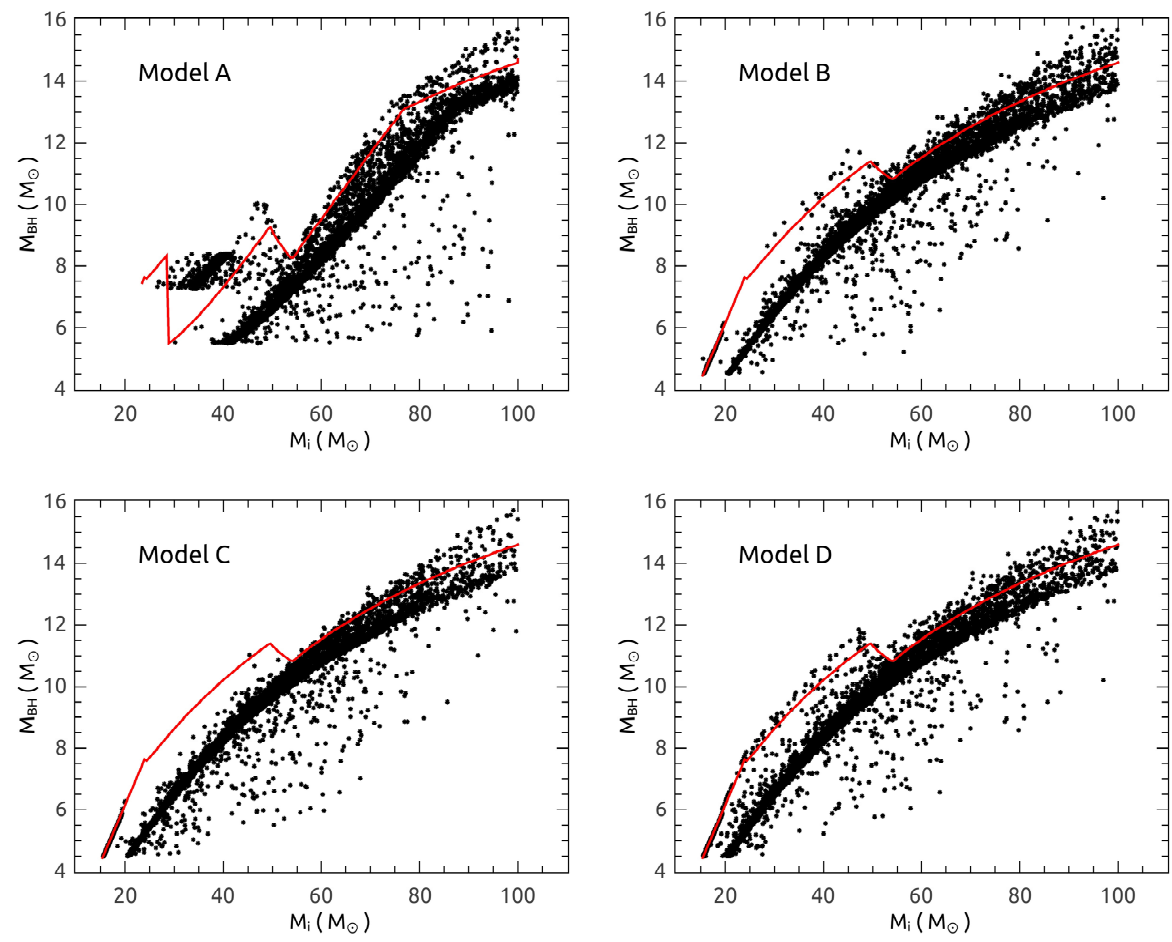

FIG. 1.- Distributions of BH mass $M_{\mathrm{BH}}$ as a function of the initial mass $M_{\mathrm{i}}$ of the primary star. The four panels correspond to Models A-D. In each panel, the black dots (with a number of $5 \times 10^{3}$ ) represent the output of the BPS calculations, and the red curve corresponds to the $\mathrm{BH}$ mass spectrum for single star evolution.

TABLE 1

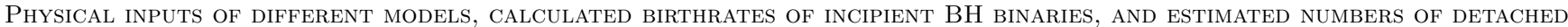
BH binaRies With MS OR Giant COMPANiONS in the GalaXy.

\begin{tabular}{cccccc}
\hline Models & $M_{\mathrm{BH}}$ & $V_{\mathrm{k}}$ & $R_{\mathrm{birth}}\left(\mathrm{yr}^{-1}\right)$ & $N_{\mathrm{BH}-\mathrm{MS}}^{*}$ & $N_{\mathrm{BH}-\mathrm{G}}^{*}$ \\
\hline $\mathrm{A}$ & $M_{\text {proto }}+M_{\mathrm{fb}}$ & $\propto 1-f_{\mathrm{fb}}$ & $9.0 \times 10^{-5}$ & $470(260)$ & $2.4(1.7)$ \\
$\mathrm{B}$ & $M_{\mathrm{rem}}$ & $\propto 3.0 / M_{\mathrm{BH}}$ & $6.0 \times 10^{-5}$ & $4100(340)$ & $160(11)$ \\
$\mathrm{C}$ & $M_{\mathrm{rem}}$ & $\sigma_{\mathrm{k}}=150 \mathrm{~km} \mathrm{~s}^{-1}$ & $4.5 \times 10^{-5}$ & $4300(285)$ & $175(12)$ \\
$\mathrm{D}$ & $M_{\mathrm{rem}}$ & $\sigma_{\mathrm{k}}=50 \mathrm{~km} \mathrm{~s}^{-1}$ & $1.3 \times 10^{-4}$ & $12000(926)$ & $595(48)$ \\
\hline
\end{tabular}

* The numbers of detached BH binaries with normal-star companions brighter than 20 mag are given in parenthesis.

low-mass secondaries are able to survive CE evolution. When applying other kick velocity distributions to the newborn BHs in Models $\mathrm{C}$ and $\mathrm{D}$, the distributions of incipient $\mathrm{BH}$ binaries are similar in the $M_{\mathrm{c}}-P_{\text {orb }}$ plane as in Model B, with the birthrates of $\sim 4.5 \times 10^{-5} \mathrm{yr}^{-1}$ and $\sim 1.3 \times 10^{-4} \mathrm{yr}^{-1}$, respectively.

In Figure 3 we plot the obtained birthrates of the incipient $\mathrm{BH}$ binaries as a function of the companion mass, $\mathrm{BH}$ mass, orbital period and eccentricity in Models $\mathrm{A}-\mathrm{D}$. Besides no low-mass companions, Model A predicts that the $\mathrm{BH}$ masses have a peak distribution at $\sim 7-8 M_{\odot}$, and the binary systems tend to have nearly circular orbits. The reason is that most of $\mathrm{BHs}$ are formed through direct collapse without a natal kick. In the other three models, the incipient $\mathrm{BH}$ binaries unsurprisingly have similar parameter distributions. Note that the binaries containing lighter $\mathrm{BHs}$ tend to have higher birthrates due to the IMF. In the following, we will simulate the Galactic population of detached $\mathrm{BH}$ systems with normal-star companions, by tracking the evolution of the incipient
$\mathrm{BH}$ binaries until the companions fill their corresponding Roche lobes. For clarification, we only show the Galactic population of detached $\mathrm{BH}$ binaries in Models A and B, the calculated results for all models are summarised in Table 1.

\subsection{Overall population of detached $B H$ binaries}

A detached BH binary can be discovered through observation of the optical companion. Based on the BPS calculations, we can predict the distributions of some observational parameters including the apparent magnitude $m_{\mathrm{V}}$, the radial velocity semi-amplitude $K$ and the astrometric signature $\alpha$. We use the stellar mass, surface luminosity and effective temperature of the optical companions to yield the absolute magnitude $M_{\mathrm{V}}$ and the stellar color $B-V$. By taking into account the interstellar extinction $A_{\mathrm{V}}$ in the $\mathrm{V}$-band, the apparent magnitude $m_{\mathrm{V}}$ is given as

$$
m_{\mathrm{V}}=M_{\mathrm{V}}+5\left(2+\log D_{\mathrm{kpc}}\right)+A_{\mathrm{V}}\left(D_{\mathrm{kpc}}\right),
$$



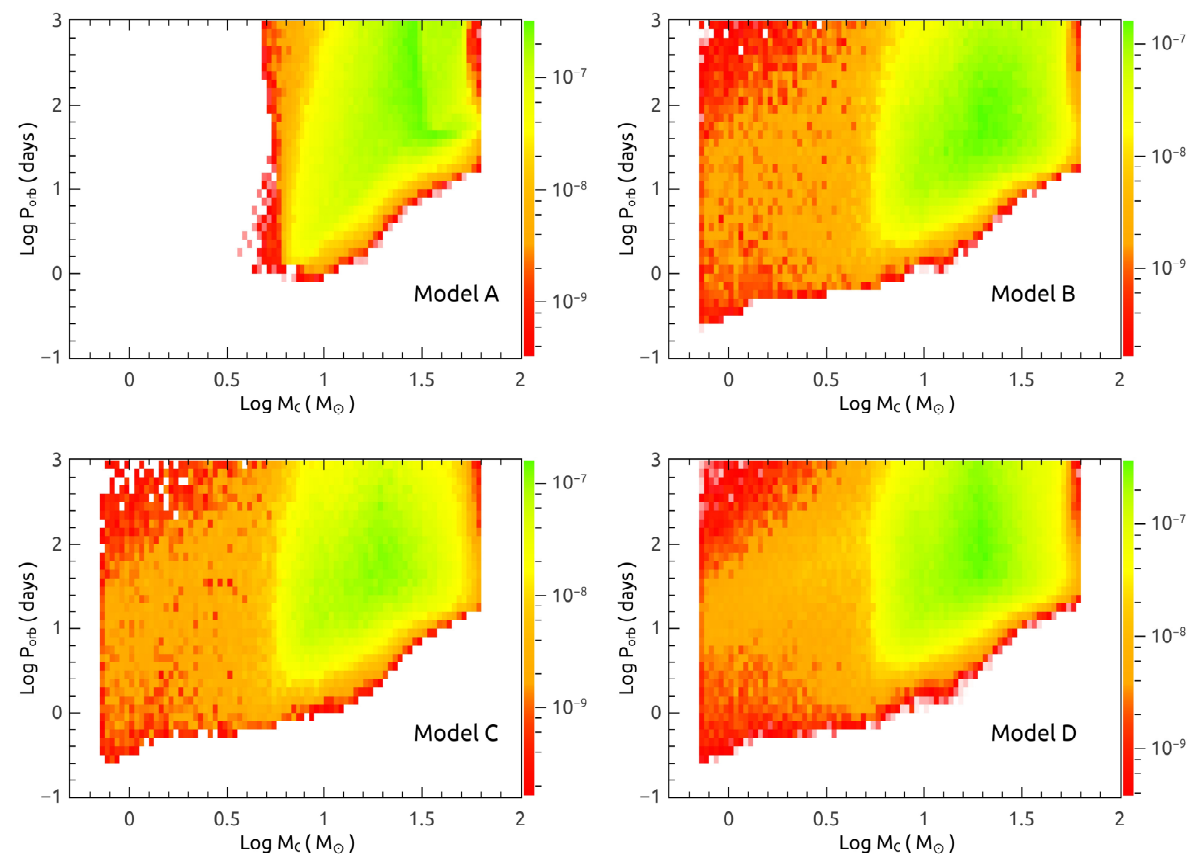

FIG. 2.- The birthrate distributions of incipient BH binaries in the companion mass $\left(M_{\mathrm{c}}\right)$ vs. orbital period $\left(P_{\text {orb }}\right)$ plane, by assuming a constant star formation rate of the Galaxy to be $3 M_{\odot} \mathrm{yr}^{-1}$. The four panels correspond to different assumed models, the colors in each pixel are scaled according to the weight of the corresponding birthrates.
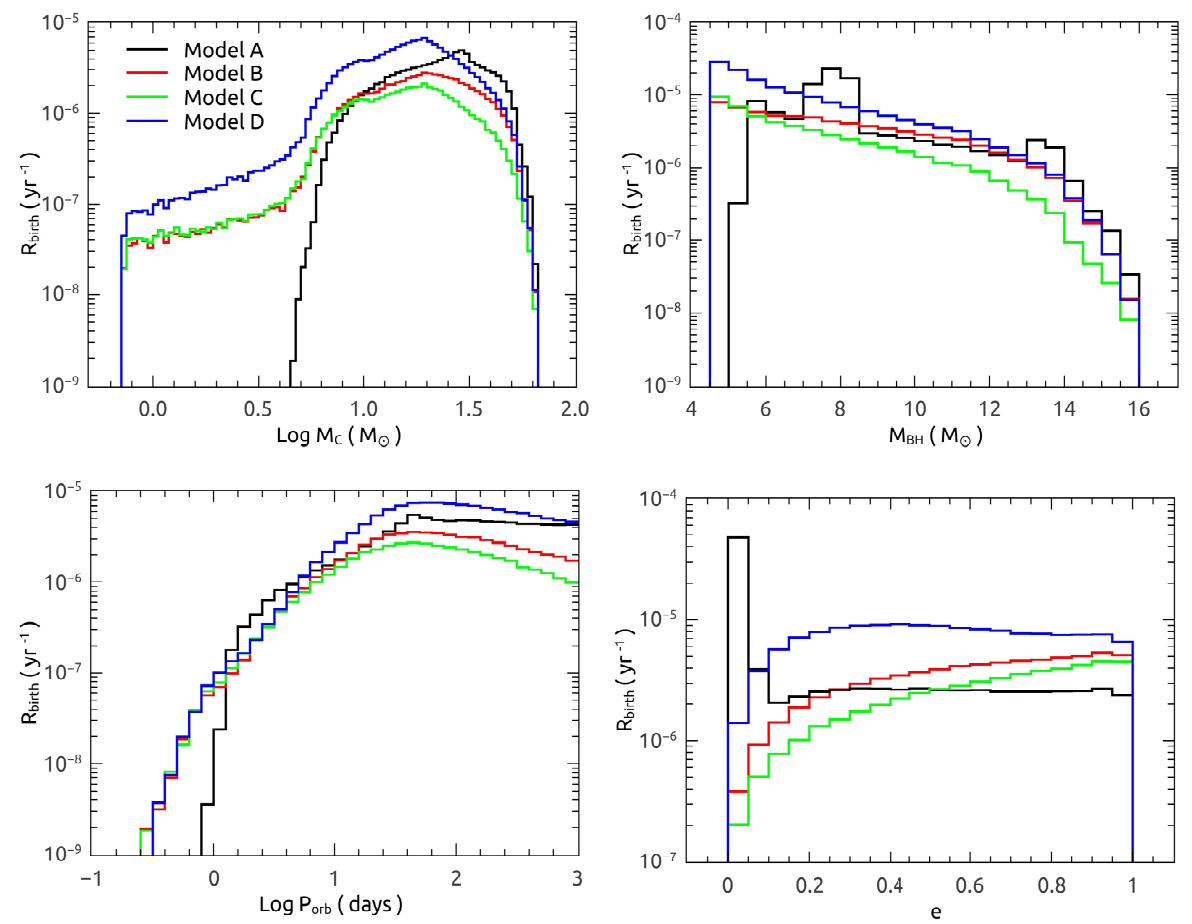

FIG. 3.- The birthrate distributions of incipient $\mathrm{BH}$ binaries in the Galaxy, as a function of companion mass $M_{\mathrm{c}}$, $\mathrm{BH}$ mass $M_{\mathrm{BH}}$, orbital period $P_{\text {orb }}$ and eccentricity $e$. The colored curves correspond to different assumed models. 
where $D_{\mathrm{kpc}}$ is the distance of the binary from the Sun normalized by $1 \mathrm{kpc}$ and we assume $A_{\mathrm{V}}\left(D_{\mathrm{kpc}}\right)=D_{\mathrm{kpc}}$ (see also Yamaguchi et al. 2018, Yalinewich et al. 2018). The radial velocity semi-amplitude $K$ of the optical companions is

$$
K=\sqrt{\frac{G}{\left(1-e^{2}\right)}}\left(M_{\mathrm{c}}+M_{\mathrm{BH}}\right)^{-1 / 2} a^{-1 / 2} M_{\mathrm{c}} \sin i,
$$

where $G$ is the gravitational constant, $e$ the eccentricity, $a$ the semi-major axis and $i$ the orbital inclination of detached BH binaries with respect to the Sun. The orientation of binary systems is assumed to have a random distribution. The astrometric signature $\alpha$ is given by

$$
\alpha=\frac{a_{\text {project }}}{D},
$$

where $D=D_{\mathrm{kpc}} \times 1 \mathrm{kpc}$, and $a_{\text {project }}$ denotes the projected semi-major axis of the companion's orbits (see also Breivik et al. 2017, Mashian \& Loeb 2017).

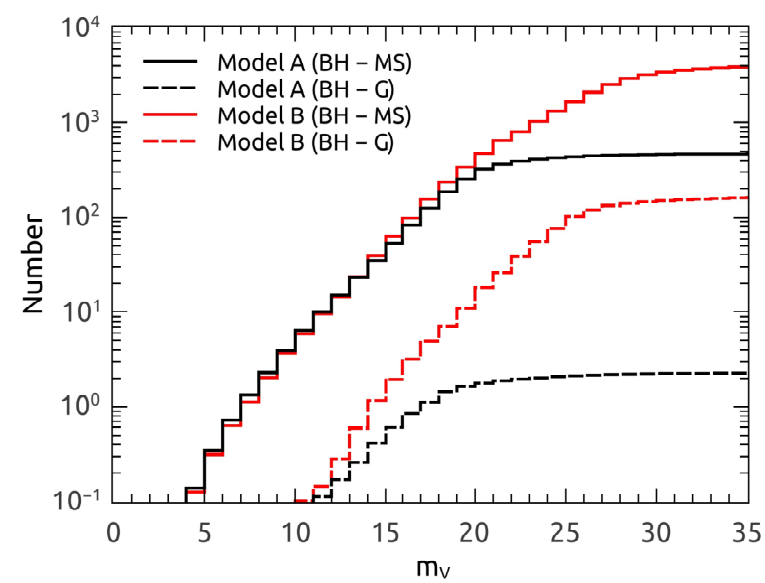

FIG. 4.- Accumulated number distributions for detached BH binaries with normal-star companions in the Galaxy as a function of apparent magnitude $m_{\mathrm{V}}$, when gradually increasing the $m_{\mathrm{V}}$ by an interval of $1 \mathrm{mag}$. The black and red curves correspond to Models A and B, and the solid and dashed curves denote the systems with MS and giant companions, respectively.

In Figure 4 we show the cumulative number distributions of detached $\mathrm{BH}$ binaries in the Galaxy when gradually increasing $m_{\mathrm{V}}$ by an interval of $1 \mathrm{mag}$. The black and red curves denote the results under the assumptions of Models A and B, and the solid and dashed curves correspond to the systems with $\mathrm{MS}$ and giant companions, respectively. We obtain that there are $\sim 500$ detached $\mathrm{BH}$ binaries in Model $\mathrm{A}$ and the number rises to $\sim 4000$ in Model B. The BH systems with MS companions dominate the overall population, only a few percent of them have giant companions.

Figure 5 presents the calculated number distributions of all detached BH binaries in the Galaxy, as a function of the orbital period $P_{\text {orb }}$, eccentricity $e, \mathrm{BH}$ mass $M_{\mathrm{BH}}$, companion mass $M_{\mathrm{c}}$, astrometric signature $\alpha$ and radial velocity semi-amplitude $K$. The black and red curves correspond to Models A and B, and the solid and dashed curves denote the binaries containing MS and giant companions, respectively. In both models, the or- bital periods distribute in a wide range of $\sim 1-1000$ days and quite a number of systems have nearly circular orbits. In addition, the $K$ distributions have a peak at $\sim 30-100 \mathrm{kms}^{-1}$ and $\alpha$ broadly distributes in the range of $\sim 3-1000 \mu$ as. The main differences between Models A and B are the component masses: the companion masses are $\gtrsim 5 M_{\odot}$ and the $\mathrm{BH}$ masses cluster $\sim 7-8 M_{\odot}$ in Model A, while both the companions and the BHs tend to have low masses (peaked at $\sim 1 M_{\odot}$ and $\sim 5 M_{\odot}$ respectively) in Model B.

\subsection{Detectable population of detached BH binaries}

Due to the limitation of astronomical instruments, the very dark companions in detached $\mathrm{BH}$ binaries cannot be detected at present. Based on current high performance of Gaia satellite with a limiting magnitude of 20 mag in the $\mathrm{G}$ band, we further discuss the detectable population of detached $\mathrm{BH}$ binaries in the Gaia era. Following Yamaguchi et al. (2018) and Yalinewich et al. (2018), we equate the Gaia band with the V band for the optical companions. For testing the valid of this assumption, we have used the color-color transformations of Jordi et al. (2010) to compute the Gaia G magnitude, and find that the population size of detached $\mathrm{BH}$ binaries is increased by a factor of only a few percent. In Figure 6 we show the number distributions of detached $\mathrm{BH}$ binaries with optical companions brighter than 20 mag in the Galaxy, under the assumptions of Models A (black curves) and B (red curves). The solid and dashed curves correspond to the systems with MS and giant companions, respectively. We can see that a large number of binaries with lowmass companions are hidden and undetected, the number of detectable binaries drops to several hundreds in both models. We emphasize that there are still over 100 detectable detached $\mathrm{BH}$ binaries with companion masses less than $\sim 5 M_{\odot}$ in Model B.

Figures 7 and 8 present the number distributions of detectable detached $\mathrm{BH}$ binaries in the $P_{\mathrm{orb}}-e$ (left panels), $M_{\mathrm{BH}}-M_{\mathrm{c}}$ (middle panels) and $\alpha-K$ planes (right panels) in Models A and B, respectively. The top and bottom panels correspond to the systems with MS and giant companions, respectively. Each panel contains $40 \times 40$ matrix elements, the colors reflect the number of detached $\mathrm{BH}$ binaries in the corresponding matrix element by accumulating the product of the birthrates with the time durations. Due to tidal interactions, the systems with $P_{\text {orb }} \lesssim 3$ days tend to have circular orbits if the companions are still MS stars, while most of binaries can be circularized if the companions have climbed to the giant branches. For the component masses, Model B particularly predicts that the low-mass $\left(\lesssim 3 M_{\odot}\right)$ normalstars are usually orbited by light $\left(\sim 4.5-6.5 M_{\odot}\right)$ BHs. There is a tendency that the larger the $\alpha$, the longer the $a$, thus the smaller the $K$.

Recently several groups (Mashian \& Loeb 2017, Breivik et al. 2017, Yamaguchi et al. 2018; Yalinewich et al. 2018) have explored the prospect of discovering BHs in binaries by Gaia based on the motions of normal-star companions. Mashian \& Loeb (2017) estimated the number of $\mathrm{BH}$ binaries that can be detected by Gaia over its five year mission to be nearly $2 \times 10^{5}$. However, they did not consider the effects of some important factors such as interstellar extinction, $\mathrm{BH}$ natal kicks and mass transfer process during binary evolution. Breivik et al. (2017) 

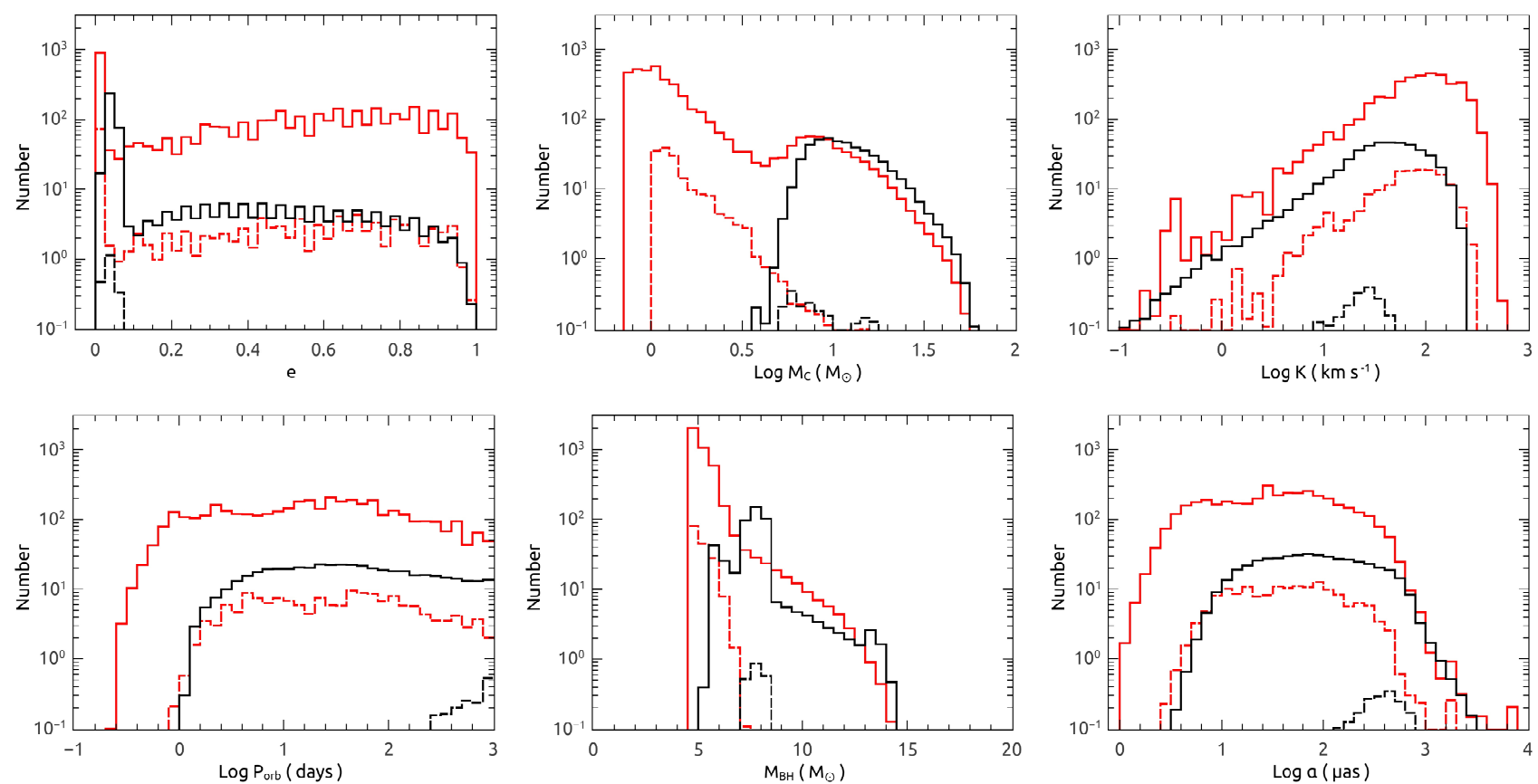

FIG. 5. - Expected number distributions of all detached BH binaries in the Galaxy, as a function of orbital period $P_{\text {orb }}$, eccentricity $e$, companion mass $M_{\mathrm{c}}$, BH mass $M_{\mathrm{BH}}$, radial velocity semi-amplitude $K$ and astrometric signature $\alpha$. The black and red curves correspond to Models A and B, and the solid and dashed curves denote the systems with MS and giant companions, respectively.
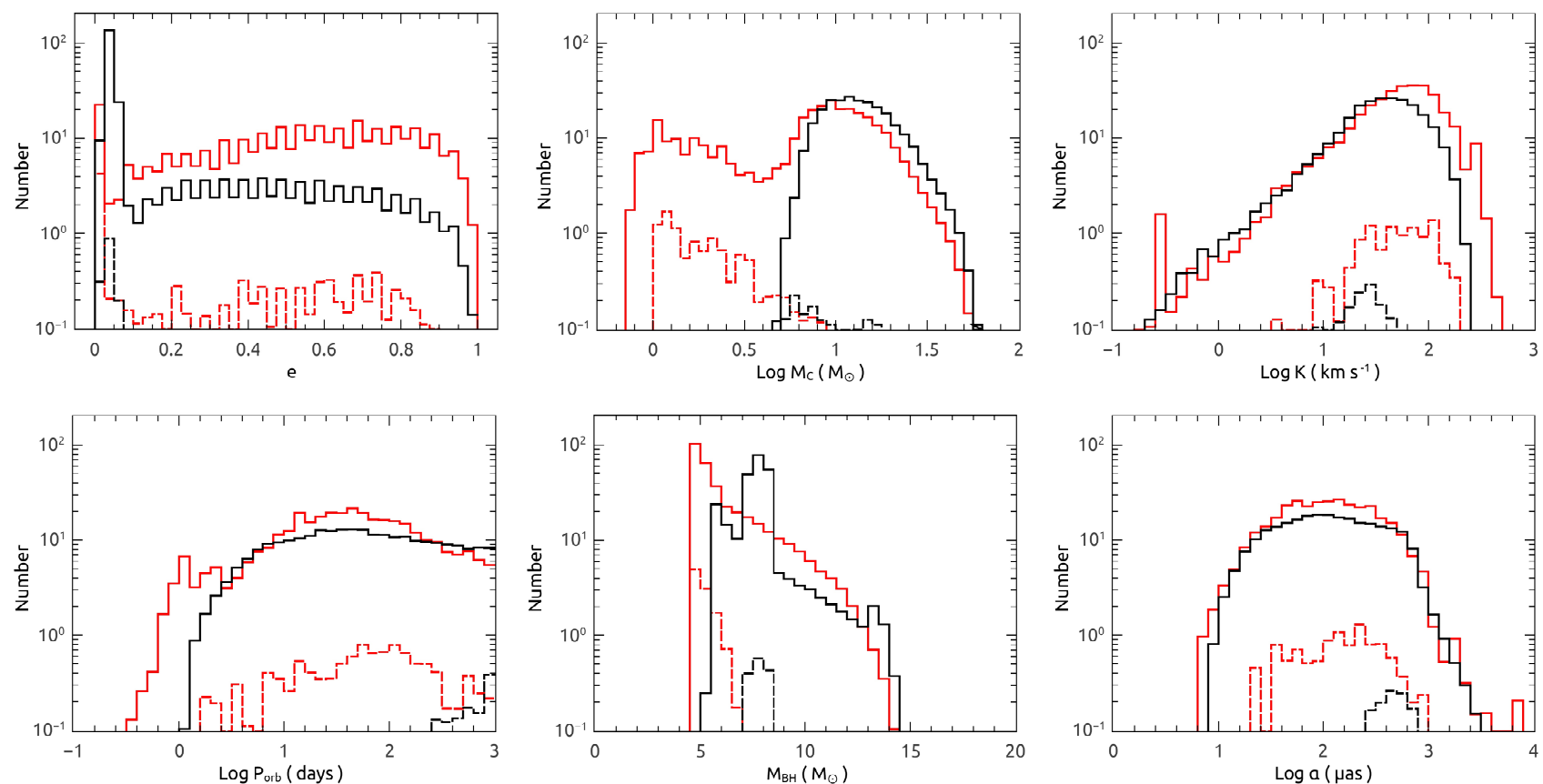

Fig. 6.- Similar to Figure 5, but for detectable BH binaries with optical companions brighter than 20 mag.

reduced the number to be $3800-12000$ by taking into account detailed treatments relevant for the formation of $\mathrm{BH}$ binaries, but the effect of interstellar extinction was still not incorporated. Yamaguchi et al. (2018) added the effect of interstellar extinction, and the estimated number of detectable BH binaries significantly decreased to $\sim 200-1000$. Furthermore, Yalinewich et al. (2018) showed that their models yield only dozens of detectable $\mathrm{BH}$ binaries with luminous companions.
When comparing our obtained population of detectable $\mathrm{BH}$ binaries with that by Yamaguchi et al. (2018) and Yalinewich et al. (2018), we need to point out that there are big differences in the treatment of the formation processes of the binary systems. (1) Before the formation of the $\mathrm{BH}$ binaries, the progenitor systems experience either $\mathrm{CE}$ evolution or stable mass transfer phases. During the CE phases, both Yamaguchi et al. (2018) and Yalinewich et al. (2018) adopted a constant 

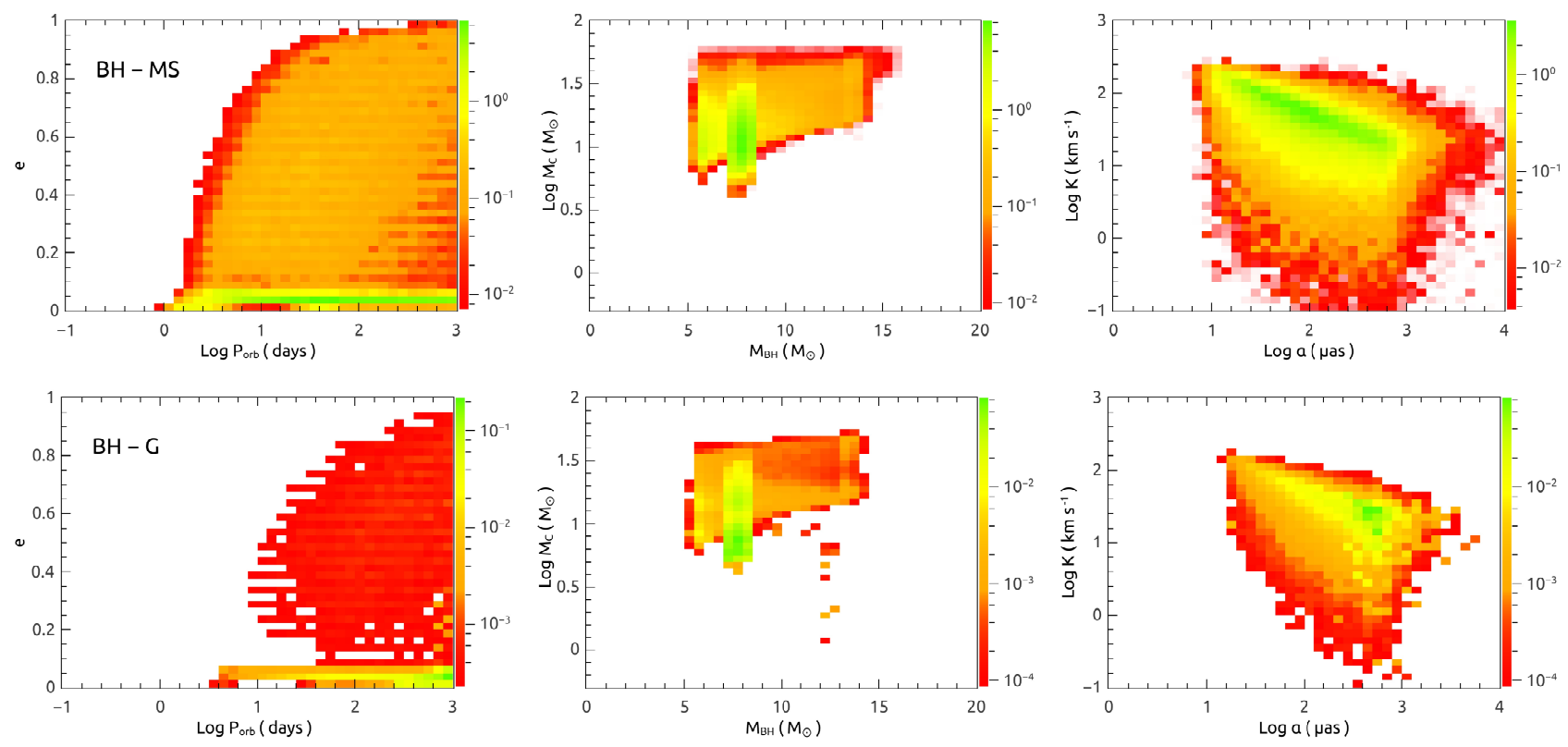

FIG. 7.- Predicted number distributions of detectable detached BH binaries in Model A. The left, middle and right panels correspond to the systems distributing in the $P_{\mathrm{orb}}-e, M_{\mathrm{BH}}-M_{\mathrm{c}}$ and $\alpha-K$ planes, respectively. The top and bottom panels correspond to the systems with MS and giant companions, respectively. The colors in each pixel are scaled according to the number of detached $\mathrm{BH}$ binaries.
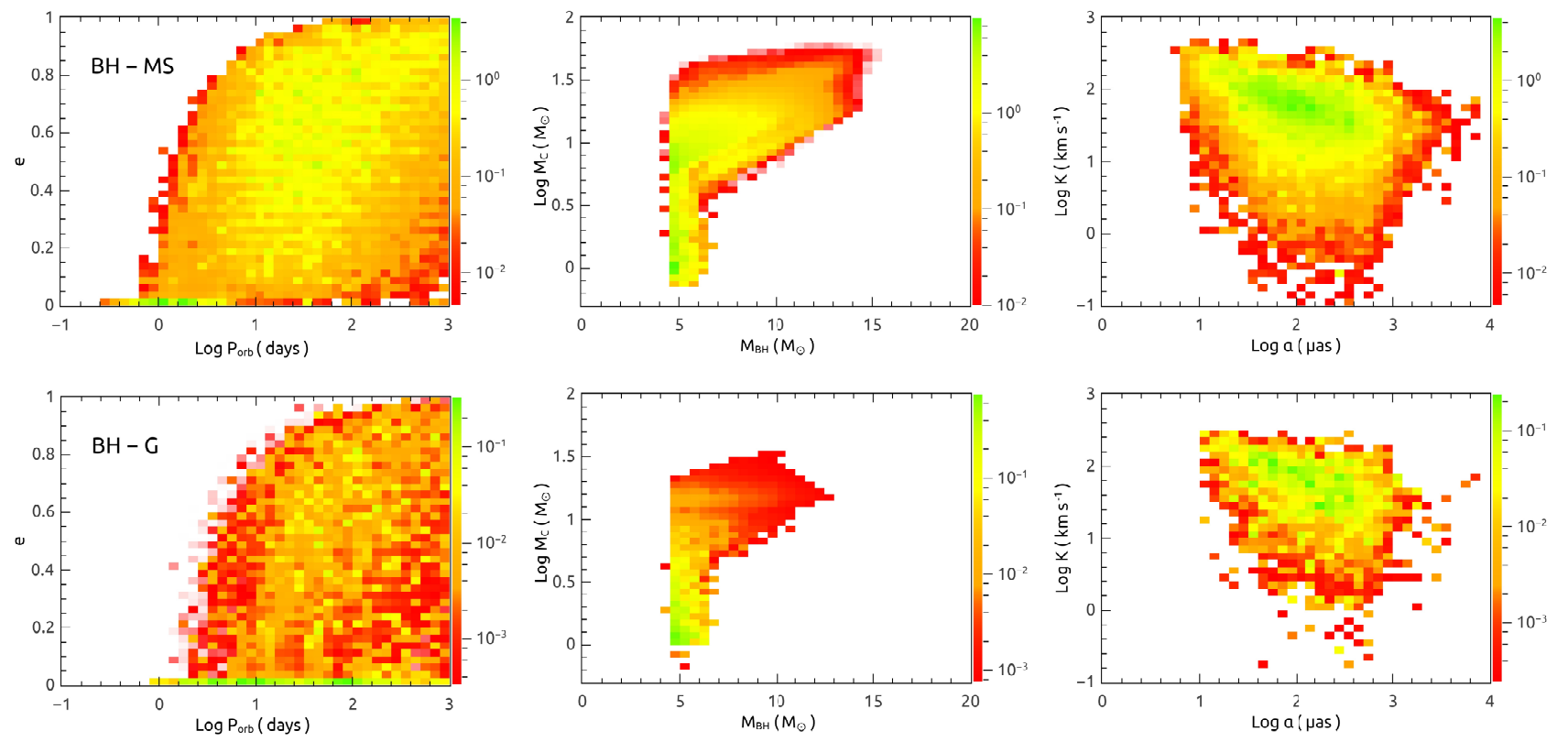

Fig. 8.- Similar to Figure 7, but in Model B.

$\alpha_{\mathrm{CE}} \lambda(0.1$ or 1.0$)$ for the CE parameter in their BPS calculations. The $\mathrm{BH}$ systems with low-mass companions could be rarely generated if taking $\alpha_{\mathrm{CE}} \lambda=0.1$, while a fraction of $\mathrm{BH}$ binaries could have low-mass companions as the survivors of $\mathrm{CE}$ evolution if taking an abnormally large value of 1.0 for $\alpha_{\mathrm{CE}} \lambda$, because numerical calculations show that $\lambda$ is usually significantly less than 1 for supergiant stars (e.g., Dewi \& Tauris 2000; Podsiadlowski et al. 2003; Xu \& Li 2010; Wong et al. 2014; Wang et al. 2016b). In our Model $\mathrm{B}$, the initial masses of $\mathrm{BH}$ progenitors can be as low as $\sim 15 M_{\odot}$, more than $100 \mathrm{BH}$ systems with low-mass companions can be created af- ter CE evolution. (2) Both Yamaguchi et al. (2018) and Yalinewich et al. (2018) indicated that the companion masses are heavier than $\sim 8-15 M_{\odot}$ due to mass accretion if the progenitor systems have experienced stable mass transfer phases. These are similar to our results in Model A, but we obtain less-massive companions with minimal masses of $\sim 5 M_{\odot}$ since the rotation-dependent mass transfer mode is adopted during primordial binary evolution. Compared to previous works, we further provide the characteristics of $\mathrm{BH}$ binaries with giant companions. Recently the discovery of a candidate $\mathrm{BH}$ orbited by a low-mass giant (Thompson et al. 2019) seems 
to require the formation channel involving a $\mathrm{CE}$ phase as proposed in our Model B.

\section{CONCLUSION}

Based on a BPS method, we have simulated the Galactic population of detached $\mathrm{BH}$ binaries with normal-star companions. Considering the uncertainties in $\mathrm{BH}$ formation physics and relevant natal kicks, we build four different models to explore the possible effects on the binary population. Model A involves the traditional theory of $\mathrm{BH}$ formation. In this model the initial masses of $\mathrm{BH}$ progenitors are $\gtrsim 25 M_{\odot}$, and the primordial binaries with a low-mass secondary cannot survive the spiral-in phases when they go into $\mathrm{CE}$ evolution. The $\mathrm{BH}$ progenitor masses can drop as low as $\sim 15 M_{\odot}$ in the other models, and this allows the formation of $\mathrm{BH}$ binaries with a low-mass companion. The predicted number of detached $\mathrm{BH}$ binaries in Model A are about one order of magnitude lower than those in other models. When only changing the $\mathrm{BH}$ natal kicks in Models B, C and D, the calculated numbers vary by a factor of less than 3 . In addition, there may exist some extra factors that can influence the potential number of detached $\mathrm{BH}$ binaries. We assume all stars are in binaries, while observations show that about $70 \%$ massive stars are actually in binary systems (Sana et al. 2012), this will slightly reduce the population size. We adopt a constant star formation rate of $3 M_{\odot} \mathrm{yr}^{-1}$ in the calculations. Many groups (e.g., Smith et al. 1978; Diehl et al. 2006; Robitaille \& Whitney 2010) obtained the star formation rate of the Galaxy varying in the range of $\sim 1-5 M_{\odot} \mathrm{yr}^{-1}$, and the actual rate is subject to slight variations along the Galactic age (Rocha-Pinto et al. 2000). These can also change the calculated number by a factor of a few.

Considering that Model A cannot produce detached $\mathrm{BH}$ binaries with low-mass companions, we summarise our main results from the other models except Model A as follows.

1. The birthrates of incipient $\mathrm{BH}$ binaries in the Galaxy are in the range of $4.5-13 \times 10^{-5} \mathrm{yr}^{-1}$ when considering different natal kick distributions for newborn BHs. The systems with companion masses larger than $\sim 5 M_{\odot}$ dominate the incipient $\mathrm{BH}$ binaries, and the birthrate of the binaries with low-mass $\left(\lesssim 5 M_{\odot}\right)$ companions is of the order $10^{-6} \mathrm{yr}^{-1}$. These two groups are roughly separated by the evolution of primordial binaries experiencing either stable mass transfer or a CE phase.

2. The overall population of detached $\mathrm{BH}$ binaries are dominated by the systems with relatively low-mass companions. We predict that the total number is over 4000 . If only considering the systems with companions brighter than 20 mag (i.e., observable by Gaia), the number of detached $\mathrm{BH}$ binaries reduces to about several hundred.

3. Our calculations show that more than 100 detached $\mathrm{BH}$ systems with giant companions in the Galaxy and among them at least 10 could be detected. Such binaries tend to have companions of mass $\lesssim 5 M_{\odot}$ and $\mathrm{BHs}$ of mass $4.5-7 M_{\odot}$, whose features are consistent with those of the candidate $\mathrm{BH}$ binary with a giant companion recently discovered by Thompson et al. (2019).

We thank the referee for useful suggestions that helped improve this paper. This work was supported by the Natural Science Foundation of China (Nos. 11973026, 11603010, 11773015, and 11563003) and Project U1838201 supported by NSFC and CAS, the National Program on Key Research and Development Project (Grant No. 2016YFA0400803), and the Natural Science Foundation for the Youth of Jiangsu Province (No. BK20160611).

\section{REFERENCES}

Abt, H. A. 1983, ARA\&A, 21, 343

Bolton, C. T. 1972, Natuer, 235, 271

Breivik, K., Chatterjee, S., \& Larson, S. L. 2017, ApJL, 850, L13

Brown, G. E., \& Bethe, H. A. 1994, ApJ, 423, 659

Burrows, A., Hayes, J., \& Fryxell, B. A. 1995, ApJ, 450, 830

Casares, J. \& Jonker, P. G. 2014, Spa. Sci. Rev., 183, 223

Dewi, J. D. M., \& Tauris, T. M. 2000, A\&A, 360, 1043

Diehl, R., Halloin, H., Kretschmer, K., et al. 2006, Nature, 439, 45

Ertl, T., Janka, H.-T., Woosley, S. E., Sukhbold, T., \& Ugliano, M. 2016, ApJ, 818, 124

Fragos, T., Andrews, J., Ramirez-Ruiz, E., et al. 2019, arXiv: 1907.12573

Fryer, C., Belczynski, K., Wiktorowicz, G., et al. 2012, ApJ, 749, 91

Giesers, B., Dreizler, S., Husser, T.-O., et al. 2018, MNRAS, 475, L15

Gu, W. M., Mu, H. J., Fu, J. B. et al. 2019, ApJ, 872, L20

Guseinov, O. K., \& Zel'dovich, Y. B. 1966, SvA, 10, 251

Hamann, W.-R., Koesterke, L., \& Wessolowski, U., 1995, A\&A, 299, 151

Hobbs, G., Lorimer, D. R., Lyne, A. G., \& Kramer, M. 2005, MNRAS, 360, 974

Hurley, J. R., Pols, O. R., \& Tout, C. A. 2000, MNRAS, 315, 543

Hurley, J. R., Tout, C. A., \& Pols, O. R. 2002, MNRAS, 329, 897

Ivanova, N., Justham, S., Chen, X., et al. 2013, A\&AR, 21, 59

Jordi, C., Gebran, M., Carrasco, J. M., et al. 2010, A\&A, 523, 48

Khokhlov, S. A., Miroshnichenko, A. S., Zharikov, S. V., et al. 2018, ApJ, 856, 158

Kiel, P. D., \& Hurley, J. R., 2006, MNRAS, 369, 1152

Kochanek, C. S., 2014, ApJ, 785, 28
Kroupa, P., Tout, C. A., \& Gilmore, G. 1993, MNRAS, 262, 545

Mapelli, M., \& Giacobbo, N. 2018, MNRAS, 479, 4391

Mashian, N., \& Loeb, A. 2017, MNRAS, 470, 2611

Masuda, K., \& Hotokezaka, K. 2018, arXiv: 1808.10856

O'Connor, E., \& Ott, C. D. 2011, ApJ, 730, 70

Pejcha, O., \& Thompson, T. A. 2015, ApJ, 801, 90

Podsiadlowski P., Rappaport S., \& Han Z., 2003, MNRAS, 341, 385

Raithel, C. A., Sukhbold, T., \& Özel, F. 2018, ApJ, 856, 35 Remillard, R. A., \& McClintock, J. E. 2006, ARA\&A, 44, 49

Robitaille, T. P., \& Whitney, B. A. 2010, ApJL, 710, L11

Rocha-Pinto, H. J., Scalo, J., Maciel, W. J., \& Flynn, C. 2000, A\&A, 358, 869

Sana, H., de Mink, S. E., de Koter, A., et al. 2012, Science, 337, 444

Smith, L. F., Biermann, P., \& Mezger, P. G. 1978, A\&A, 66, 65

Shao, Y., \& Li, X.-D. 2014, ApJ, 796, 37

Shao, Y., \& Li, X.-D. 2016, ApJ, 833, 108

Shao, Y., \& Li, X.-D. 2018, ApJ, 867, 124

Spera, M., Mapelli, M., \& Bressan, A. 2015, MNRAS, 451, 4086

Sukhbold, T., Ertl, T., Woosley, S. E., Brown, J. M., \& Janka, H.-T. 2016, ApJ, 821, 38

Thompson, T. A., Kochanek, C. S., Stanek, K. Z., et al. 2019, arXiv: 1806.02751

Timmes, F. X., Woosley, S. E., \& Weaver, T. A. 1996, ApJ, 457, 834

Trimble, V. L., \& Thorne, K. S. 1969, ApJ, 156, 1013

Ugliano, M., Janka, H.-T., Marek, A., \& Arcones, A. 2012, ApJ, 757,69 
van den Heuvel, E. P. J. (1992), in ESA, Environment Observation and Climate Modelling Through International Space Projects (SEE N9323878 0888)

Vink, J. S., de Koter, A., \& Lamers, H. J. G. L. M. 2001, A\&A, 369,574

Wang, C., Jia, K., \& Li, X.-D. 2016a, MNRAS, 457, 1015

Wang, C., Jia, K., \& Li, X.-D. 2016b, RAA, 16, 126

Webbink, R. F. 1984, ApJ, 277, 355

Webster, B. L., \& Murdin, P. 1972, Nature, 235, 37
Wong, T.-W., Valsecchi, F., Ansari, A., et al. 2014, ApJ, 790, 119 Woosley, S. E., \& Weaver, T. A., 1995, ApJS, 101, 181

Xu, X.-J., \& Li, X.-D. 2010, ApJ, 716, 114

Yalinewich, A., Beniamini, P., Hotokezaka, K., \& Zhu, W. 2018, MNRAS, 481, 930

Yamaguchi, M. S., Kawanaka, N., Bulik, T., \& Piran, T. 2018, ApJ, 861, 21

Yungelson, L. R., \& Lasota, J.-P., 2008, A\&A, 488, 257 